\title{
Application of Open CNC System in NC Machine Reform
}

\author{
Yong-Hua Huang ${ }^{1,}$ a and Han gao ${ }^{2}$ \\ ${ }^{1}$ Department of mechanical engineering, Shandong vocational college of science and technology, \\ Weifang City Shangdong Province, zip code 261053,China \\ ${ }^{2}$ School of computer engineering, Weifang University, Weifang City Shangdong Province, zip code \\ 261061, China \\ ahuangyonghua@126.com
}

Keywords: CNC Machines; Open CNC system; PC machine

\begin{abstract}
With the increasing development and improvement of numerical control technology, the users put forward higher requirements of the machine's intelligent, multi-functional and flexible. More flexible choice and configuration software and hardware has become the inevitable trend of numerical control technology in the condition of different use of the environment and requirements to achieve the open transformation of CNC Machines. It mainly studies the development trend of the numerical control system, the open system of motion control card and PC unit core, while puts forward the concrete application of the open CNC system and the specific characteristics of the open CNC Machine.
\end{abstract}

\section{Introduction}

With the rapid development of science and technology, and the increasing development of the secondary industry In recent years, CNC Machines is being more perfect showing high flexibility, high efficiency, high precision, high integration and so on. In order to improve the overall competitiveness of the products, machine tool manufacturers require the controller to be open, with greater compatibility, thereby improving the performance of the controller, so it is necessary for the transformation of CNC Machines to use open CNC system

\section{Open CNC system and its advantages}

Open CNC system. Open CNC system appeared in the 90's of 20th century, fully integrated with the computer hardware and software, control technology and information technology. The so-called open system refers to an open system should enable a variety of applications to effectively run on different providers to provide the platform, with other applications and interoperability of users and the characteristics of the exchange [1]. Because of its portability, openness and interoperability and other characteristics, so even if the hardware manufacturers are different, can also be produced in accordance with uniform standards to provide hardware platform. Different system software can be installed on the hardware platform and run to form the corresponding system platform, and processing enterprises can according to their needs to select all kinds of operating systems, so as to rebuild the different hardware and software model, to meet their own needs to build the NC system. These different function modules can support certain standard file formats and communication protocols, replaces, coordinates, and calls each other.

Advantages of open CNC system. The traditional machine tool adopts the universal numerical control system, which is bad specialty and strong versatility, the system manufacturer master the software core, making the software update, the support and the function expansion inconvenience, at the same time for some external software that are poor suppression and ability, while the machine tool manufacturers and operators of the operational level of the higher requirements, it has a greater limitation [2]. In this study, the PC machine as a basis for the transformation of CNC Machines, making open CNC system scalability, portability, interchangeability, multi-tasking, scalability, network, interoperability Sex, special, etc. can be met, which is the previous general controller cannot 
match, the obvious advantages, to a large extent to meet the urgent need for machine tool manufacturers open requirements. The rapid development and gradually mature open CNC system in recent years, under the environment of PC market competition, the production producers want to win the competitive advantage, and constantly improve the cost-effective PC, promote rapid development of the PC-based open CNC system in a large extent.

\section{Structural characteristics of open CNC system}

Currently the common open CNC system on the market set the movement control card and PC as the core, and the ISA / PCI bus, STD bus and other forms of the floor as a template, accordance with the specific needs of the system to expand [3]. Specifically, the open CNC system mainly includes the hardware structure and software results, as follows.

Hardware structure. Open CNC system compared with the previous general CNC system, has a significant advantage in scalability, scalability, flexibility. Bus module based on the main drive control module, PC module and additional modules of the three modules, these three modules together constitute a modular, CPU open system, showing in Fig. 1.

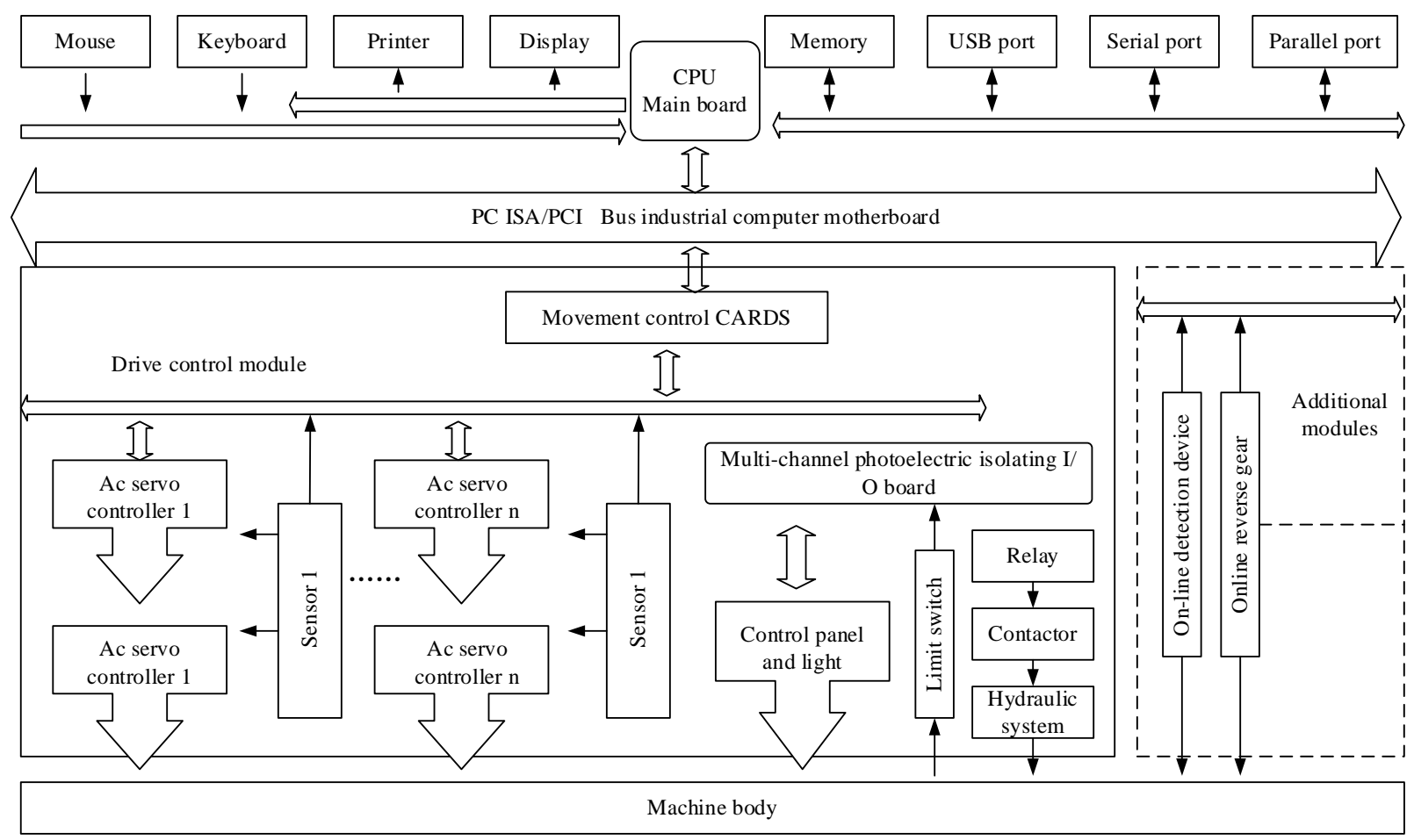

Figure 1. Open CNC system

(1) PC module. The so-called PC module refers to the host computer module, using all the advantages of PC and compatible all attachments [4]. The core components of the PC module in this study are CPU templates, which are responsible for all non-real-time processing tasks such as human-machine interface and background management. PC CPU operation is more stable, and faster, can be used in any application software or large-scale system software to run; at the same time before processing through the CPU on-site simulation test, to the degree of stability and reliability of inspection, Making the rejection rate significantly reduced [5]. Windows mode of operation interface more emotional and control, the operator is more easy to operate, while through the serial port, parallel port, Internet, USB jack and other extrapolation interface, can connect multiple hosts, to achieve the sharing of resources.

(2) Drive control module. Drive control module is the lower computer module, its core control components are motion control card, the drive control module and the CPU and other parts together to form a high-speed communication with the bus connection, and the implementation of servo control, contour processing, pitch compensation, plug Complement operation, tool radius compensation and all real-time control tasks. In general, the 32-bit digital processor handles the motion calculation of 
the motion control card and provides simplified programming and maximum program throughput [6]. Since the 90's of last century, Europe and the United States and other Western countries and Japan have been researched and develop on the open CNC system, the current foreign controller manufacturers to manufacture such control card technology has been relatively mature. Through the motion control card can be multi-axis programming and control, flexible performance. Each card can be manipulated not more than 8 axes at the same time, and these axes can be used to achieve a single axis group linkage in a coordinate system. In addition, these axes can be fully independent in their respective coordinate systems. Group movement. Although the eight axes can be operated at the same time, each axis has a relatively independent nature and can be arbitrarily combined between every two axes. Each PC can be inserted 16 such cards at the same time, which is meaning that it can convert at least 128 movement of the axis at the same time operation after the realization of open CNC system.

(3) Additional modules. Machine tool manufacturers in this module can be in accordance with their actual control needs to transform and add some additional special features. Such as online reverse technology, online testing, robot and automatic loading and unloading agencies. Conventional controllers used in the past have not been allowed to add or change these functions privately, and an open CNC system based on a PC can allow the machine tool builder to perform an open retrofit in this piece.

Software structure. The system use the Windows operating system as an open CNC system software system, where are more advantages, can develop a variety of advanced programming language as their own needs applications. In these developed applications, the NC core system has a good definition of the export point for efficient use, and the CNC kernel system and the application of effective connection. Export point as a convergence of this way to assist the NC system to collect users to use preferences, experience, their own knowledge and special technology, so that the NC system and user needs fit more well, becoming a unique dedicated system, where is more obvious advantage of targeted and reliability in some specific Occasions.

\section{Application of Open CNC System in CNC Machine Reform}

The CNC Machines using open CNC system makes the system more flexible and open. Special CNC spiral milling machine is mainly used for all kinds of special-shaped screw processing, requiring the machine to achieve four-axis movement in $\mathrm{X}, \mathrm{C}, \mathrm{Z}, \mathrm{A}$, and the four axis are driven by four AC servo motor bear. In order to make the accuracy of the machine more accurate, Z-axis with a grating, to form a fully closed-loop position control; in order to eliminate the interference with other axes to meet the different lead and head number. Different spiral angle screw processing requirements, A-axis servo motor to drive the main control of the swing angle, so that the motor more accurate. Open CNC system, the same coordinate system of the five-axis linkage, the NC system to achieve the processing state of the milling head swing, the work piece rotation movement, the tool for the work piece axial movement, radial movement and its own rotation control, So that between different instruments to complete the precise, complex spatial interpolation movement [7]. The tool edge and the work piece helical surface form the 'meshing' between the point and point so that the helical surface of the entire work piece is covered by the envelope. CNC Machine processing is mainly elongated screw, the longest work piece length reached $8 \mathrm{~m}$, the tool in the processing process will inevitably wear and tear, which led to the work piece error. In order to reduce this error, it can be tool compensation and on-line detection to reduce the error in the processing process, so that improving the accuracy of processing significantly.

Through the high-precision grating digital displacement sensor to form a closed measurement chain, open CNC system deal with the collected data responsibly, and in accordance with the final results of the tool to compensate for the need to determine the value of the tool. In addition, the screw surface is not a smooth plane, but the complex surface, so the current algorithm cannot be applied to all the data points on the surface to calculate and include, mainly through the coordinate measuring machine to measure, so the measurement results cannot be used directly. Through the combination of 
coordinate measurement technology and numerical control system, it is possible to shorten the development cycle of the new product program as much as possible by reversing this function online. The numerical control system integrated of the above new technology and hardware has the advantages of small size, compact structure, high degree of modularity, large storage capacity, convenient system maintenance and upgrade [8].

The open CNC system software is applied mainly through the upper and lower computer communication driver, the host computer user applications and monitoring the variety of input and output of the PLC program. When the customers have special needs, it can be used the program to write the program forwardly, and in accordance with the need to install or uninstall the software, the machine has nothing to do with the useless features to delete, more prominent its dedicated features to make it in some small CNC system can also Convenient use. Through this function can be large-scale general-purpose CNC system to delete the idle function, making the NC machine tool CNC system structure more scientific and specific features.

In this system application, it combine organically the interference determination, cutting process simulation, no instantaneous core enveloping screw milling technology, error compensation technology, precision analysis, parametric programming and SCAM programming, so that complex screw processing programming can be more simple. With the visualized software interface, the user input the relevant data in accordance with the interface prompts, which can quickly get the desired effect in a short time. Through the output graphics and simulation data, it can automatically generate high efficiency, high precision screw processing procedures, in response to complex procedures can also be quickly generated in a short time, effectively reducing the preparation process required for the auxiliary work time. Through the open system, it can realize the machine alarm prompt automatically, spindle speed five adjustment, machine linkage control, friendly user interface design, program management, graphical and modular, that the general CNC system is completely unmatched. The Open CNC system manufacturers have provided the upper and lower machine communication, and classify processing and package the main function of the, the user does not need to reuse the preparation. PLC program mainly contains the system automatic function, each manual function, the spindle movement control, mainly for the machine input, the control panel as well as the output control.

In the preparation process of the PLC program, I/O port and DPRAM address mirroring, I/O port state is stored in DPRAM, so PLC machine and motion control card for information exchange, and only need to share the same storage space can, can realize the user-defined communication function of the system, manual and automatic the main way, storyand reverse, state information feedback, users on the processing state of real-time monitoring.

In the preparation of the PLC program, do the mirror of the I/O port and DPRAM address, that the I/O port state stored in the DPRAM, so it only need to share the same storage space when exchange the information between the PLC machine and the motion control card, to achieve user-defined communication function, system manual and automatic mode, spindle positive and reverse, the feedback of status information, real-time monitoring of processing for users, et al..

\section{Conclusion}

PC is the basis of open CNC system, responsible for the support of speed control, position control, interpolation and other real-time tasks assisted by the powerful motion control card. Compared with the traditional CNC system, it was realized the multi-faceted open, human-computer interaction and the integration of more powerful, so help users and machine tool manufacturers to re-customize, develop and upgrade the system, making the system more practical and practical ,having a broad vision of application. 


\section{References}

[1] Li Lingxiao, Lu Qiangzhong. An open-architecture PC-based programmable multi-axis controller (PMAC) [J]. Mechatronics, 2002, (1): 41-44.

[2] Li Xueguang, Sui Lihui, Chen Lidan, et al. Design of CGM6125 Machine Tool Based on Open CNC System [J]. Machine Tool \& Hydraulics, 2016,44 (4): 1-5.

[3] Lu Xiaohu, Yu Dong, Hu Yi, et al. Research on embedded numerical control system based on heterogeneous multi-core processor [J]. China Mechanical Engineering, 2013,24(19): 2623-2628.

[4] Su Chun, Chen Zhiwei, Cui Pengfei, et al. A Case Study of CNC Remanufacturing of Old Machine Tool [J]. Mechanical Design and Manufacturing Engineering, 2014,43 (1): 46-49.

[5] Cao Huajun, Du Yanbin, Zhang Mingzh, et al. Machine tool remanufacturing and comprehensive upgrading of the connotation and technical framework [J]. China Surface Engineering, 2010,23 (6): 75-79.

[6] Fu Hongya, Hu Po, Han Dedong. Research Progress of STEP-NC Numerical Control Technology [J] .Computer Integrated Manufacturing System, 2014,20 (3): 569-578.

[7] Sun Liping, Zou Xuan. MC1332/H cylindrical grinding machine numerical control transformation program and implementation [J]. Machine Tool \& Hydraulic, 2014,42(14): 165-168,161.

[8] Li Lingxiao, Lu Qiangzhong. An open-architecture PC-based programmable multi-axis controller (PMAC) [J]. Mechatronics, 2002 (1): 41-44. 